\title{
Intersection of ChIP and FLIP, genomic methods to study the dynamics of the cohesin proteins
}

\author{
Adrian J. McNairn • Jennifer L. Gerton
}

Published online: 20 March 2009

(C) The Author(s) 2009. This article is published with open access at Springerlink.com

\begin{abstract}
The evolutionarily conserved cohesin proteins Smc1, Smc3, Rad21 (Mcd1), and Scc3 function in the cohesin complex that provides the basis for chromosome cohesion and is involved in gene regulation. Understanding how these proteins link together the genome requires the use of wholegenome approaches to study the molecular mechanisms of these essential proteins. While chromatin immunoprecipitation followed by DNA microarray (ChIP-chip) studies have provided a snapshot in time of where these proteins associate with various genomes, the cohesin proteins are dynamic in their localization and interactions on chromatin. Study of the dynamic nature of these proteins requires approaches such as live cell imaging. We present evidence from fluorescence loss in photobleaching (FLIP) experiments in budding yeast that the decay constant of each cohesin subunit is $\sim 60-90 \mathrm{~s}$ in interphase. The decay constant on chromatin increases from $G_{1}$ to $S$ phase to metaphase, consistent with the interaction with chromatin becoming more stable once chromosomes are cohered. A small population of Smc3 at a position consistent with centromeric location has a longer decay constant than
\end{abstract}

Responsible Editor: Christian Haering.

A. J. McNairn · J. L. Gerton $(\bowtie)$

Stowers Institute for Medical Research, 1000 E. 50th Street,

Kansas City, MO 64110, USA

e-mail: JEG@stowers.org bulk Smc3. The characterization of the interaction of cohesin with chromatin, in terms of both its position and its dynamics, may be key to understanding how this protein complex contributes to chromosome segregation and gene regulation.

Keywords cohesin $\cdot$ FLIP $\cdot$ cell cycle $\cdot$ SMC
Abbreviations
Abd-B abdominal-B
ChIP chromatin immunoprecipitation
CTCF CCCTC-binding factor
DAPI 4',6-diamidino-2-phenylindole dihydrochloride
EGFP enhanced green fluorescent protein
FLAG epitope tag
FLIP fluorescence loss in photobleaching
FRAP fluorescence recovery after photobleaching
FRET fluorescence resonance energy transfer
Mcd1 mitotic chromosome determinant 1
Pds5 precocious dissociation of sisters
Scc sister chromatid cohesion
SMC structural maintenance of chromosomes

\section{Introduction}

From the time the chromosomes replicate until they separate at the metaphase-to-anaphase transition, sister chromatids are held together by a cohesive force. This cohesion is achieved via a protein 
complex known as the cohesin complex. The complex consists of four subunits, Smc1, Smc3, Scc3 (SA1, SA2), and Mcd1/Scc1 $(\operatorname{Rad} 21)$, which are thought to form a ring that can hold sister chromatids together (Haering et al. 2008; Onn et al. 2008). However, many other proteins have been shown to be important for chromosome cohesion, including those involved in loading the cohesin complex onto DNA (Scc2, Scc4), those which couple cohesion to DNA replication (Eco1), those involved in maintaining cohesion (Pds5), and those involved in dissolving cohesion (separase, polo-like kinase). The collective activity of all of these factors can be thought of as the cohesin pathway. Most components of this pathway are evolutionarily conserved from budding yeast to human.

The question of where cohesin proteins localize within the genome is of foremost importance, as the binding of the cohesin complex to chromatin is critical for multiple cellular processes including chromosome cohesion, DNA damage repair, and proper gene regulation. The chromosomal sites at which cohesin associates with DNA have been mapped by ChIP-chip (chromatin immunoprecipitation followed by hybridization to DNA microarrays) in yeast, flies, and humans (Glynn et al. 2004; Lengronne et al. 2004; Misulovin et al. 2008; Parelho et al. 2008; Stedman 2008; Wendt et al. 2008) and by in-situ chromosome staining (McGuinness et al. 2005; Gomez et al. 2007). These studies revealed both similarities as well as disparities in the localization of cohesin proteins among different eukaryotes. One similarity is that centromeric regions show high levels of cohesin association, which presumably is important for chromosome segregation. Cohesin binding sites are also found throughout the chromosome arms spaced semi-periodically and can exhibit cell-specific chromosomal localizations that correlate with the epigenetic state of the chromatin. In budding yeast, cohesin is largely found in regions of convergent transcription and is absent from actively transcribed genes (Glynn et al. 2004; Lengronne et al. 2004). In contrast, fly cohesin binds preferentially to transcribed regions and overlaps with RNA polymerase II (Misulovin et al. 2008). In mammalian cells, most sites are in intergenic regions and introns (Wendt et al. 2008).

A number of observations suggest that although cohesin can reproducibly associate with the same regions of the genome each time it loads onto chromosomes, it does not recognize a specific sequence. In flies and yeast, there has been no motif significantly associated with the bound sequences (Glynn et al. 2004; Lengronne et al. 2004; Misulovin et al. 2008). In humans, cohesin binding along chromosome arms correlates with a consensus sequence that has been previously identified as the CTCF binding site (Parelho et al. 2008; Stedman et al. 2008; Wendt et al. 2008). CTCF is a conserved enhancer blocking protein found in vertebrates and is associated with a number of functions, including transcription, X-chromosome inactivation, and imprinting (Wallace and Felsenfeld 2007). The ability of CTCF to induce intra- and inter-chromosomal interactions may create regions of juxtaposed DNA to which cohesin may bind and stabilize. However, when CTCF is depleted, cohesin still appears to associate with DNA, but its binding is significantly delocalized, arguing that the site specificity is provided by the CTCF protein (Wendt et al. 2008). Thus, as is the case in lower eukaryotes, the cohesin complex itself is able to associate with DNA without sequence specificity in higher eukaryotes.

The position of the cohesin complex on DNA is altered under different gene expression programs. In yeasts, cohesin in chromosome arms is found predominantly in intergenic regions between convergent transcription units (Glynn et al. 2004; Lengronne et al. 2004). However, there are also some open reading frames where cohesin is found, of which some are inducible genes. When these genes are induced by heat shock, addition of galactose, or amino acid starvation, cohesin no longer localizes to these regions (Glynn et al. 2004; Lengronne et al. 2004; Bausch et al. 2007). Furthermore, when transcription is repressed, cohesin can return (Bausch et al. 2007). In flies, cohesin binds to the Abd-B homeobox gene in cells in which it is transcribed, but not in cells in which it is silenced (Misulovin et al. 2008). These results emphasize the lack of specificity for any particular DNA sequence, but also highlight that cohesin association with DNA is dynamic, depending on transcription.

The ChIP-chip experiments strongly suggest that the association of cohesin subunits with DNA is dynamic. The dynamics of the complex have been suggested to be controlled by a variety of proteins such as the maintenance subunit Pds5 (Losada et al. 
2005) and the loading factor Scc2 (Rollins et al. 1999, 2004). For instance, in $X$. laevis egg extracts, mitotic chromosomes assembled in the absence of Pds5 have intact arm cohesion, but centromeric cohesion is loosened, although chromosomes retain an unusually high level of cohesin (Losada et al. 2005). Thus, Pds5 may play both positive and negative roles in sister chromatid cohesion, possibly by directly modulating the dynamic interaction of cohesin with chromatin. Alterations in the dynamics of the complex as caused by mutation of Scc2 have been suggested to affect the transcription of the cut locus in flies (Rollins et al. 2004). Understanding the dynamics of the complex may prove to be critical for understanding how the complex contributes to gene regulation.

Chromatin immunoprecipitation is a powerful method for gaining a high-resolution snap shot of where a protein of interest is located. The association of cohesin with a particular sequence can be followed at the resolution of a few hundred base pairs over a timescale of minutes to hours. However, this method is not optimal for capturing the behavior of a protein of interest on a very short timescale, such as seconds to minutes. Also, it measures where a protein of interest is located or enriched in a population of cells, but does not allow single cell measurements. A complementary approach to ChIP-chip experiments is live-imaging experiments. In these types of experiments, a protein of interest is fused to a fluorescent protein and the properties of the fusion protein can be assessed through bleaching the fluorescence with a laser and measuring how the protein behaves. These studies have the advantage of being able to monitor the behavior of a protein in a single cell over seconds to minutes (Rabut and Ellenberg 2005; Sprague and McNally 2005).

In order for sister chromatid cohesion to persist from the time sisters are replicated until mitosis, the linkage provided by cohesin would have to be stable over this period of time. However, many experiments have shown that cohesin can change locations, suggesting that its association with chromatin can be dynamic. In addition, a large fraction of cohesin on chromosomes arms dissociates during prophase in mammalian cells, while cohesin at the centromere remains until the metaphase-to-anaphase transition. In order to address how cohesin associates with chromatin during the cell cycle, Gerlich and colleagues performed a study in which a cohesin subunit, Scc3/ SA1, was fused to EGFP and monitored by photo- bleaching and quantitative live-imaging by iFRAP (inverse fluorescence recovery after photobleaching) in the rat NRK cell line (Gerlich et al. 2006). They found three subpopulations of Scc3: (1) about 50\% of Scc3 is soluble and highly dynamic, (2) about 30 $50 \%$ is chromatin-bound with an exchange rate of $16 \mathrm{~min}$, and (3) a fraction of $10-20 \%$, present only in late $S$ and $G_{2}$ phase of the cell cycle, appears to be stably bound to chromatin, with an exchange rate of greater than $1 \mathrm{~h}$ (Gerlich et al. 2006). They speculate that the stably bound pool mediates sister chromatid cohesion. Consistent with this proposal, Bloom and colleagues have found evidence for a very stable cylindrical array of Smc3 in mitotic yeast nuclei that they propose organizes pericentric chromatin into an intramolecular loop (Yeh et al. 2008).

Since its discovery, cohesin has been studied intensively in budding yeast due to the ease of manipulation and the many tools available in this model organism. We have explored the dynamics of each cohesin subunit (Mcd1, Smc1, Smc3, and Scc3) and the accessory proteins Pds5 and Scc2 using fluorescence loss in photobleaching (FLIP) in budding yeast. Each protein was fused to GFP and its properties were monitored in interphase. We find that each subunit has a decay constant on the order of 1$1.5 \mathrm{~min}$. The decay constant for Smc1 increases 4fold from $\mathrm{G}_{1}$ phase to $\mathrm{S}$ phase to M-phase, consistent with the stability of the association with DNA increasing over the course of the cell cycle. Finally, a pool of Smc3 with slower kinetics may correspond to pericentric cohesin. These studies provide evidence that cohesin subunits are able to exchange on and off chromatin and potentially in and out of the complex in vivo on a timescale of much less than a cell cycle without the loss of chromosome cohesion.

\section{Materials and methods}

GFP-tagged yeast strains were obtained from the yeast GFP collection (Open Biosystems; Thermo Fisher Scientific, Huntsville, AL, USA). A dilution growth assay was used to determine whether the tagged proteins resulted in any defects in cell growth. For immunoprecipitation, whole-cell extracts were prepared as previously described (Camahort et al. 2007). Strains expressing Smc1-GFP, Smc1-GFP and Smc3-FLAG, Smc1-GFP and Mcd1-FLAG were 
utilized. The FLAG tags were introduced into the GFP strains using homologous recombination and standard transformation procedures. Immunoprecipitations were carried out overnight at $4{ }^{\circ} \mathrm{C}$ using antiFLAG M2 antibody (Sigma, St Louis, MO, USA). Immunocomplexes were precipitated using protein $\mathrm{G}$ agarose beads (GE Biosciences). Western blots were probed with an anti-GFP antibody (Clontech) to verify the expression of a full-length protein. The Smc3-GFP strain was transformed with a plasmid expressing Spc42-RFP (a gift from R. Li) in order to obtain the image in Fig. 3.

In fluorescence loss in photobleaching (FLIP) experiments, a single spot within the nucleus with a diameter of $0.2-0.5 \mu \mathrm{m}$ was repeatedly bleached with one iteration of $100 \%$ power of a $488 \mathrm{~nm}$ laser line (50\% output) using a Zeiss LSM510 Meta with 100X PlanFluor lens. The average diameter of a single $z-$ axis plane of the yeast nucleus is $\sim 2-2.5 \mu \mathrm{m}$, resulting in approximately $10 \%$ of the nucleus being bleached with each pulse. Yeast strains were plated onto synthetic complete agar pads in hemi-spherical depression slides. Twenty pre-bleach images were collected and then images were captured before and after each bleach pulse, with $2.5 \mathrm{~s}$ between bleach pulses. After collecting 200 images, six separate measurements within the nucleus are taken to measure fluorescence loss (see Fig. 2B). Unbleached control cells were monitored for photobleaching due to image acquisition, and all measurements were normalized to the background fluorescence. Decay constants (the rate at which fluorescence is lost) for cohesin and control proteins were determined using OriginLab using nonlinear curve regression to a single exponential decay.

For cell cycle experiments, logarithmically growing yeast strains were synchronized using alpha-factor to achieve a $G_{1}$-phase arrest and then released into either $0.2 \mu \mathrm{m}$ hydroxyurea for S-phase arrest or $15 \mu \mathrm{g} / \mathrm{ml}$ nocodazole for mitotic arrest.

\section{Results}

\section{In vivo kinetics}

In recent years, the development of optimized GFP variants and better microscope technology has led to the increased usage of photobleaching methods for study of the in vivo dynamics of chromatin-associated proteins. A large number of proteins have now been examined by photobleaching, demonstrating a wide variety of decay constants and differential kinetics (Phair et al. 2004). These methods have even been able to distinguish multiple populations of a tagged protein within the nucleus (Phair et al. 2004). An apparent trend seen in photobleaching experiments is that proteins involved in certain cellular processes, such as transcription or DNA replication, exhibit increased residence times when they are actively engaged (Dundr et al. 2002; Sporbert et al. 2002).

Utilizing yeast strains containing integrated Cterminal GFP fusions to cohesin proteins under the control of their endogenous promoters at their endogenous locations, we have examined the kinetics of cohesin subunit exchange by fluorescence loss in photobleaching (FLIP). In FLIP, a single spot within the nucleus is repeatedly bleached with a $488 \mathrm{~nm}$ laser and measurements of fluorescence loss from multiple points within the nucleus are measured. These measurements may then be used to approximate the decay constant of the protein of interest, which reflects the residency time. As the cohesin proteins are essential for cell viability and growth, it was first necessary to verify that the fusion proteins are functional. As shown in Fig. 1A, the C-terminal GFP tags do not affect cell growth, indicating that the tagged proteins are functional. We demonstrate for Smc1-GFP that it incorporates into a complex with Smc3 and Mcd1 by co-immunoprecipitation from whole cell extracts (Fig. 1B).

In addition to cohesin subunits, we used FLIP to examine the behavior of Dun1, a kinase involved in DNA damage response (Zhou and Elledge 1993). We examined the kinetics of the Dun1 protein to ensure that the power of the laser bleach pulse did not activate the DNA damage pathway. This is an important control, since the cohesin proteins have roles in DNA damage repair. The kinetics of Dun1 were unaffected by repeated bleach pulses, whereas the Dun1 kinetics change dramatically in response to hydroxyurea, which results in stalled forks and activation of the DNA damage response (decay constant increased from $113.54 \pm 6.61 \mathrm{~s}$ to $246.16 \pm$ $18.2 \mathrm{~s}$ ). Thus, the bleach pulse alone is insufficient to activate the DNA damage response pathway.

In logarithmically growing cells, we find that each cohesin subunit exchanges with its own unique 
a

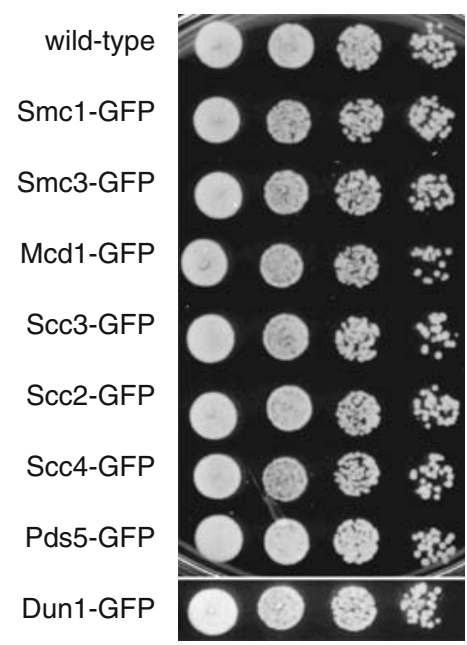

Fig. 1 GFP-tagging of proteins does not adversely affect cell growth; a As the cohesin proteins are all essential genes, it was necessary to determine whether tagging the proteins with GFP would affect cellular growth. Strains containing GFP-tagged subunits integrated at their endogenous loci and the wild-type parental strain were serially diluted and plated onto YPD. Strains were grown at $30^{\circ} \mathrm{C}$ for $48 \mathrm{~h}$. There are no differences between the GFP-tagged strains and the control, indicating that GFP-tagging does not adversely affect the functionality of the proteins; b Smc1-GFP is integrated into the cohesin complex. Strains containing Smc1-GFP (control), or Smc1-GFP with

kinetics (Table 1). On average, the rate of loss for cohesin pathway proteins was $1-1.5 \mathrm{~min}$ for interphase cells. Scc2, a component of the cohesin loading complex, and Smc3 exhibited similar kinetics, whereas Smc1 and Scc3 appeared to exchange with slower kinetics. We did not find evidence for a pool of freely diffusing cohesin subunits, which would be expected to have a recovery rate on the order of a few seconds as has been determined for nuclear GFP in budding yeast (Karpova et al. 2008). This suggests that when the cohesin proteins are expressed at their endogenous levels, the vast majority is chromatin associated.

To further examine the kinetics of cohesin subunits, the dynamics of Smc1 and Mcd1 were examined at different stages in the cell cycle (Fig. 2). The decay constant of Smc1 increases 4-fold from $\mathrm{G}_{1}$-phase (54 s) to M-phase (213 s), with an intermediate value in $\mathrm{S}$ phase (106 s). The Mcd1 subunit also exhibits an increased decay constant in M-phase (126 s) relative to interphase (Fig. 2). These increases in decay constants over the cell cycle may reflect an initial association in $G_{1}$ that is the least stable, followed by b

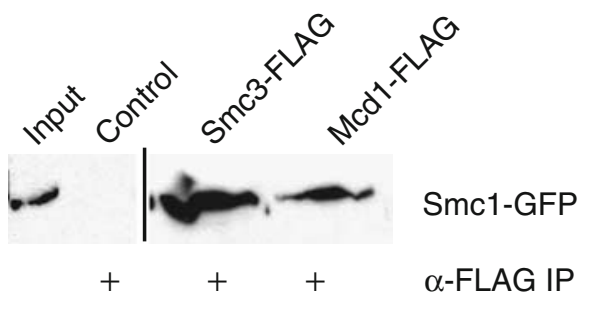

either Smc3-FLAG or Mcd1-FLAG were subjected to immunoprecipitation with anti-FLAG antibodies. The resulting immunocomplexes were then probed with an anti-GFP antibody. Smc1-GFP is specifically precipitated with either Smc3FLAG or Mcd1-FLAG, but not in a strain lacking a FLAGtagged protein, indicating it is incorporated into the cohesin complex. The input lane contains lysate corresponding to $5 \%$ of the volume subjected to immunoprecipitation. The vertical black line indicates where intervening lanes were cropped from the immunoblot

an increasingly stable association as cohesion is established in $\mathrm{S}$ phase, followed by the most stable association just prior to chromosome separation. These decay constants correlate well with the previously reported kinetics of mammalian cohesin proteins (Gerlich et al. 2006).

Table 1 Interphase kinetics of cohesin pathway proteins ${ }^{\mathrm{a}}$

\begin{tabular}{lc}
\hline GFP-tagged protein & Decay constant (s) \\
\hline Smc1 & $96 \pm 3.5$ \\
Smc3 & $55.4 \pm 1.4$ \\
Mcd1 & $67.6 \pm 1.8$ \\
Scc3 & $91.6 \pm 4.8$ \\
Scc2 & $58.7 \pm 3.3$ \\
Pds5 & $70.9 \pm 1.5$ \\
Dun1 & $113.5 \pm 6.6$
\end{tabular}

${ }^{a}$ Asynchronous cultures of the indicated GFP-tagged proteins were subjected to FLIP as described in Materials and Methods. To test interphase cells, only small budded cells were utilized, which would indicate cells in late $G_{1}$ /early $S$ phase. Each number represents the mean \pm the standard deviation. 
a

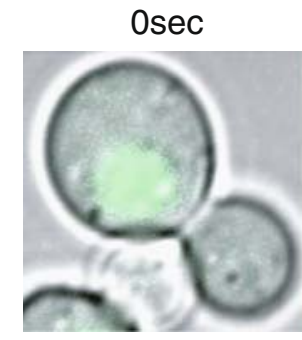

$53 \mathrm{sec}$

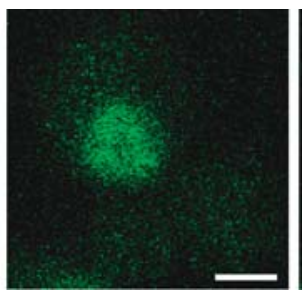

$140 \mathrm{sec}$

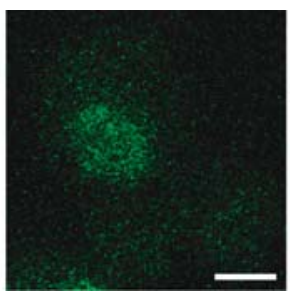

$228 \mathrm{sec}$

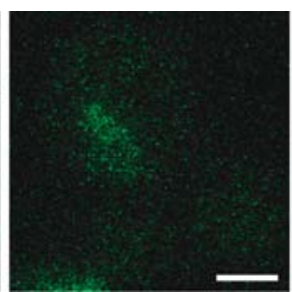

$316 \mathrm{sec}$

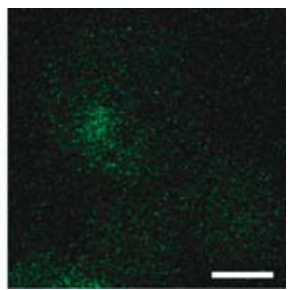

b

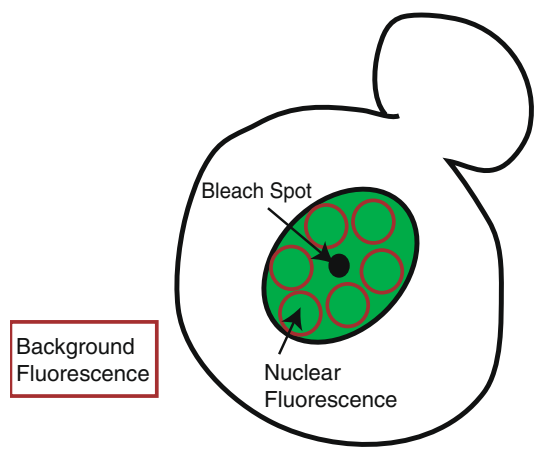

C 1.2

d

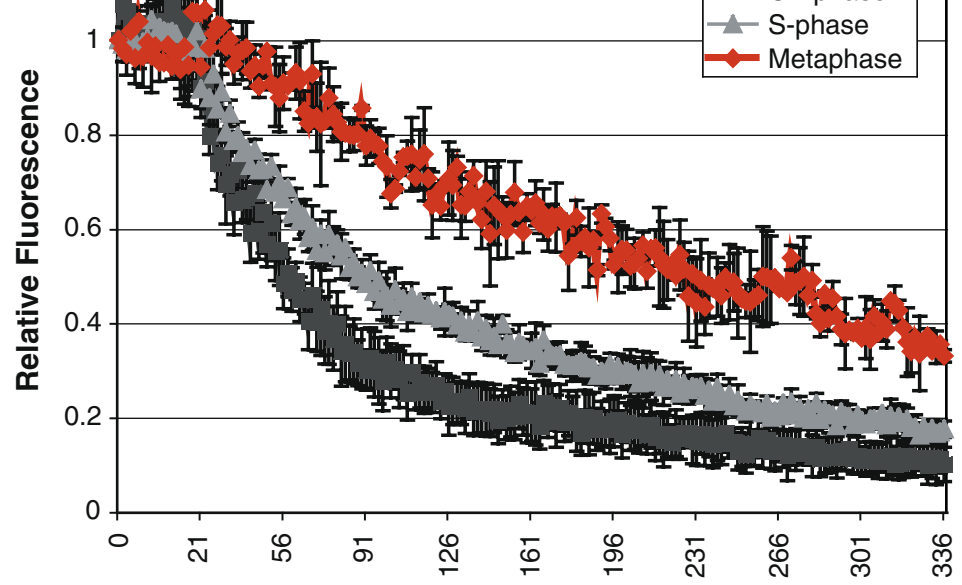

Time [sec]

\begin{tabular}{l|lll}
\hline GFP-tagged Protein & $\begin{array}{l}\text { G1-phase } \\
(\alpha \mathrm{F})\end{array}$ & $\begin{array}{l}\text { S-phase } \\
(\mathrm{HU})\end{array}$ & $\begin{array}{l}\text { Mitosis } \\
(\text { Noc })\end{array}$ \\
\hline Smc1 & $54.3 \pm 1.2$ & $106 \pm 5$ & $213 \pm 15$ \\
Mcd1 & $\mathrm{N} / \mathrm{D}$ & $\mathrm{N} / \mathrm{D}$ & $126.9 \pm 4.2$ \\
Scc2 & N/D & $89.7 \pm 4.7$ & N/D \\
Dun1 & N/D & $246.2 \pm 18.2$ & N/D \\
\hline
\end{tabular}

Fig. 2 FLIP kinetic plots of cohesin proteins; a Raw images from FLIP experiment with Smc1-GFP demonstrating the loss in fluorescence over time. The scale bar represents $2 \mu \mathrm{m}$; b Schematic diagram indicating the location of the bleach spot (filled black circle) and locations of fluorescence intensity measurements taken (open red circles); c Smc1-GFP exhibits multiple cell-cycle-dependent changes in its kinetics. Smc1GFP cells were either synchronized in $\mathrm{G}_{1}$ phase with $\alpha$-factor and analyzed, or were released into $0.2 \mu \mathrm{M}$ hydroxyurea to

Subnuclear populations of cohesin

Centromeric regions in yeast show high levels of cohesin association by ChIP-chip, whereas cohesin mapping at mammalian centromeres by ChIP has not yet been determined due to the highly repetitive alpha satellite DNA associated with mammalian centromeres. However, high levels of cohesin present at arrest them in early S-phase. For metaphase arrest, cells were first synchronized with $0.2 \mu \mathrm{M}$ hydroxyurea and released into $15 \mu \mathrm{g} / \mathrm{ml}$ nocodazole. Cells were then subjected to FLIP analysis. Lines represent averages of data collected from 20 30 cells. Error bars represent the standard deviation among the averaged results from multiple experiments; d Summary of kinetics derived from cell cycle FLIP experiments for the indicated proteins

mammalian centromeres have been observed by microscopy (McGuinness et al. 2005; Gomez et al. 2007). Cohesin at the centromere may help resist the force of microtubules until all of the chromosomes are properly aligned, and this resistance may also assist in biorientation of sister chromatids.

The enrichment of cohesin at centromeres in $S$. cerevisiae has been demonstrated both by ChIP 

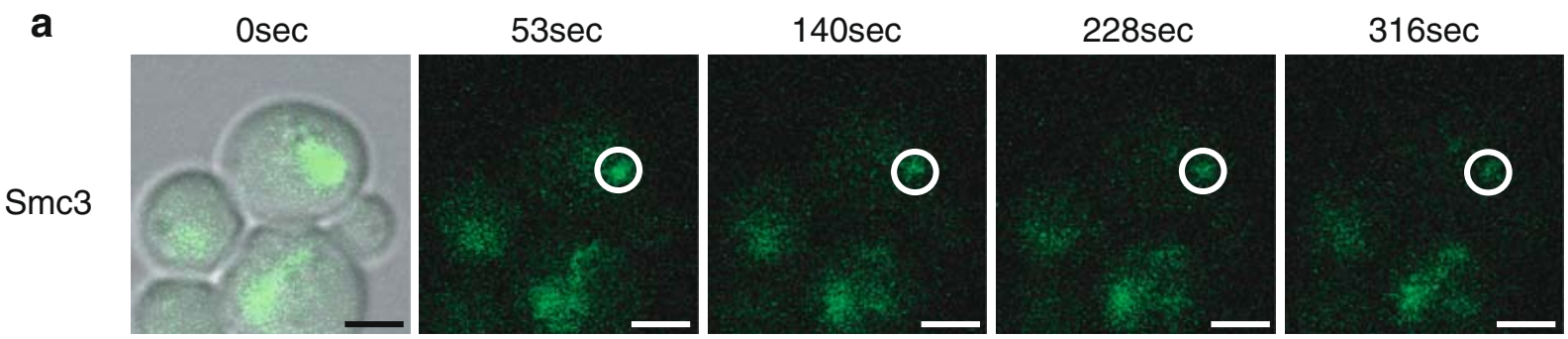

b

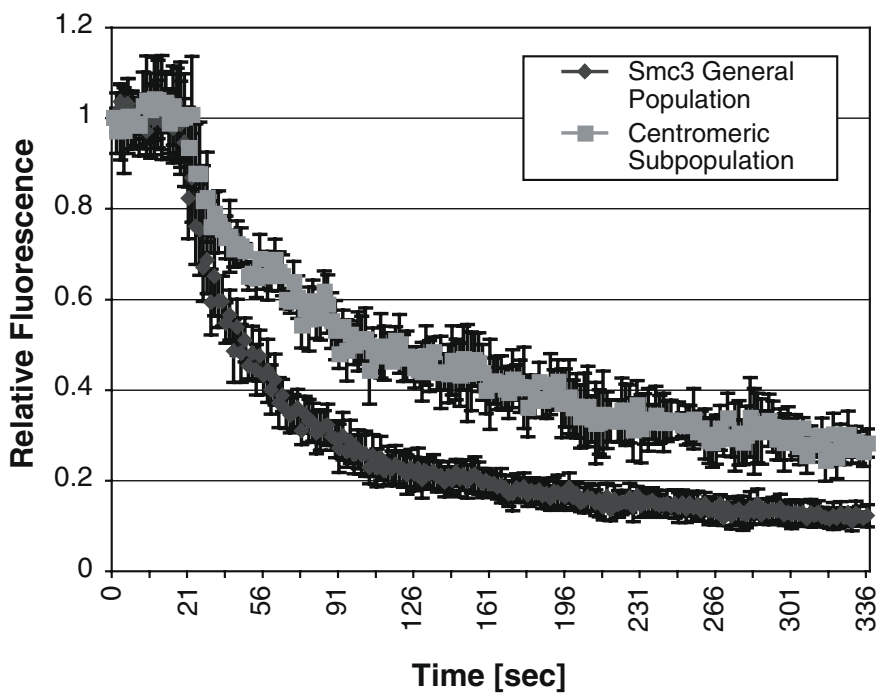

C

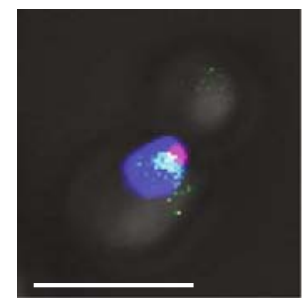

Fig. 3 A subpopulation of cohesin molecules exchange significantly more slowly than the rest of the nuclear population; a Examination of cohesin localization identified the presence of a subnuclear enrichment of cohesin that accumulates in a cell-cycle-dependent manner. Smc3-GFP is shown as an example. The white circle indicates the subpopulation of Smc3-GFP that most probably corresponds to pericentric cohesin. The scale bar represents $2 \mu \mathrm{m}$; b FLIP analysis of logarithmically growing Smc3-GFP cells deter-

methodologies and by microscopy (Weber et al. 2004; Mc Intyre et al. 2007; Yeh et al. 2008). The enrichment of cohesin around centromeres and the clustering of centromeres in yeast have enabled the visualization of cohesin subunit interactions by fluorescence resonance energy transfer (FRET) (Mc Intyre et al. 2007). In the course of our study, we found Smc3 to be enriched at a subnuclear domain in a cell-cycle-dependent manner (Fig. 3). Previously published work on the in vivo localization of Smc3, as well as the position of this population relative to the bud neck (Fig. 3A) and the spindle pole body (Fig. 3C), strongly suggests that it is pericentric (Bystricky et al. 2005; Mc Intyre et al. 2007; Yeh et al. 2008). FLIP analysis of Smc3-GFP associated with the centromeres shows that it mined that the subpopulation of Smc3 associated with the centromeres exhibits a longer decay constant than the general nuclear population of Smc3 $(55.4 \pm 1.4 \mathrm{~s}$ versus $93.2 \pm 2.4 \mathrm{~s})$; c Cells co-expressing Smc3-GFP and Spc42-RFP were arrested with nocodazole, fixed with $70 \%$ ethanol, and stained with DAPI. The DAPI signal is shown in blue, Spc42 in red, and Smc3 in green. The location of the intense $\mathrm{Smc} 3$ signal next to the spindle pole body suggests that this population is centromeric. The scale bar represents $5 \mu \mathrm{m}$

exchanges less frequently than the general population of $\mathrm{Smc} 3(55.4 \pm 1.4 \mathrm{~s}$ versus $93.2 \pm 2.4 \mathrm{~s})$ (Fig. 3B). These results confirm and extend the previous observation that cohesin can differentially associate with different subnuclear structures (Yeh et al. 2008).

\section{Discussion}

The sites at which cohesin associates with the genome are of great interest, since they will very likely correlate with local sister cohesion and/or gene regulation. The high-resolution snapshot obtained from ChIP-chip studies enables us to appreciate that 
cohesin can be bound to a particular sequence in one transcriptional program and removed under a different transcriptional program. Thus, it is likely that cohesin can respond to changes in the chromatin environment. The dynamics of the association of cohesin with chromatin has been explored using live cell imaging. Photobleaching studies in mammalian cells have demonstrated that the cohesin subunits Scc3 and Mcd1 are capable of dynamically exchanging on and off the chromatin, with a decrease in rate as the cell cycle progresses (Gerlich et al. 2006). Here we confirm and extend these results for budding yeast, demonstrating a similar increase in the stability of the Smc1 association with chromatin over the cell cycle, which correlates well with the engagement of the cohesin complex in chromosome cohesion. We have examined the decay constant for all subunits of the complex and also for two accessory factors in the cohesin pathway. For each we measured a decay constant of $\sim 1-1.5 \mathrm{~min}$, indicating the dynamic nature of these genome linker proteins. A small pool of pericentric Smc3 has slower kinetics. The picture of the cohesin pathway that emerges from the ChIP-chip and live cell imaging data suggests that neither alone will be sufficient to explain the molecular function of these proteins in gene regulation and cohesion. By combining the knowledge from these two types of experiments, a more holistic model of cohesin behavior may be elucidated.

Our results for the $S$. cerevisiae cohesin complex dynamics are mostly consistent with those previously reported for the human subunits (Gerlich et al. 2006). The immobile fraction, which they speculate corresponds to centromeric cohesin, had a residence time of several hours, or about $20 \%$ of the length of a cell cycle $(24 \mathrm{~h})$. Although we do not observe a pool of cohesin with this very long residence time, we do observe a pool of Smc3 that is less mobile and likely corresponds to pericentric cohesin. The freely diffusing population observed in the previous study, which we do not detect, may have been an artifact of the overexpression of the Scc3-GFP protein. Thus, the differential dynamics of pericentric cohesin as compared to arm cohesin, which appears to be evolutionarily conserved from yeast to human, might suggest that cohesin has different functions at these different locations. In addition, the large pool of cohesin subunits with short residence times suggests that cohesin subunits in a complex may be able to exchange, presumably without globally compromising sister cohesion.

Several factors have been identified that can alter the exchange rate of cohesin subunits. Depletion of Wapl, a protein that co-immunoprecipitates with the cohesin complex, by RNAi in HeLa cells results in an increase in the residence time of the 'fast' population of Scc1 (Kueng et al. 2006). In contrast, depletion of sororin reduces the amount of Smcl that is stably bound to chromatin (Schmitz et al. 2007). Mutations in Scc2/Nipped-B result in the localized loss of cohesin and cohesin-mediated enhancer blocking at the cut gene locus in Drosophila (Rollins et al. 2004). Mutations in Scc2 are associated with the human disease Cornelia de Lange syndrome. It has been proposed that the mutations might alter the ability of the cohesin complex to interact with chromatin, which could affect local gene regulation (Dorsett 2004). It seems likely that elucidation of both the dynamics and the location of the cohesin subunits on DNA will provide the molecular clues needed to understand this human disease.

While it seems clear that cohesin can dynamically associate with chromatin and with specific regions of chromatin, the question remains as to what chromatin features cohesin recognizes and how its association influences gene regulation. Additional types of experiments will be necessary to address these questions. The use of genome conformation capture technology in specific cell types may prove useful for discerning which non-contiguous regions of the genome might be held together by cohesin to influence gene regulation. Genetic screens may also prove useful to identify pathways that act in parallel with cohesin for gene regulation.

Acknowledgements We would like to thank Joel Schwartz, Scarlett Gard, and the Stowers Institute Imaging Center. We thank the Hudson Foundation for financial support.

Open Access This article is distributed under the terms of the Creative Commons Attribution Noncommercial License which permits any noncommercial use, distribution, and reproduction in any medium, provided the original author(s) and source are credited.

\section{References}

Bausch C, Noone S et al (2007) Transcription alters chromosomal locations of cohesin in Saccharomyces cerevisiae. Mol Cell Biol 27(24):8522-8532 
Bystricky K, Laroche T et al (2005) Chromosome looping in yeast: telomere pairing and coordinated movement reflect anchoring efficiency and territorial organization. J Cell Biol 168(3):375-387

Camahort R, Li B et al (2007) Scm3 is essential to recruit the histone h3 variant cse4 to centromeres and to maintain a functional kinetochore. Mol Cell 26(6):853-865

Dorsett D (2004) Adherin: key to the cohesin ring and cornelia de Lange syndrome. Curr Biol 14(19):R834-R836

Dundr M, Hoffmann-Rohrer U et al (2002) A kinetic framework for a mammalian RNA polymerase in vivo. Science 298(5598):1623-1626

Gerlich D, Koch B et al (2006) Live-cell imaging reveals a stable cohesin-chromatin interaction after but not before DNA replication. Curr Biol 16(15):1571-1578

Glynn EF, Megee PC et al (2004) Genome-wide mapping of the cohesin complex in the yeast Saccharomyces cerevisiae. PLoS Biol 2(9):E259

Gomez R, Valdeolmillos A et al (2007) Mammalian SGO2 appears at the inner centromere domain and redistributes depending on tension across centromeres during meiosis II and mitosis. EMBO Rep 8(2):173-180

Haering CH, Farcas AM et al (2008) The cohesin ring concatenates sister DNA molecules. Nature 454(7202):297-301

Karpova TS, Kim MJ, Spriet C, Nallcy K, Stasevich TJ, Kherrouche Z, Heliot L, McNally JG (2008) Concurrent fast and slow cycling of a transcriptional activator at an endogenous promoter. Science 319(5862):466-469

Kueng S, Hegemann B et al (2006) Wapl controls the dynamic association of cohesin with chromatin. Cell 127(5):955967

Lengronne A, Katou Y et al (2004) Cohesin relocation from sites of chromosomal loading to places of convergent transcription. Nature 430(6999):573-578

Losada A, Yokochi T et al (2005) Functional contribution of Pds5 to cohesin-mediated cohesion in human cells and Xenopus egg extracts. J Cell Sci 118(Pt 10):2133-2141

Mc Intyre J, Muller EG et al (2007) In vivo analysis of cohesin architecture using FRET in the budding yeast Saccharomyces cerevisiae. EMBO J 26(16):3783-3793

McGuinness BE, Hirota T et al (2005) Shugoshin prevents dissociation of cohesin from centromeres during mitosis in vertebrate cells. PLoS Biol 3(3):e86

Misulovin Z, Schwartz YB et al (2008) Association of cohesin and Nipped-B with transcriptionally active regions of the Drosophila melanogaster genome. Chromosoma 117 (1):89-102
Onn I, Heidinger-Pauli JM et al. (2008) Sister chromatid cohesion: a simple concept with a complex reality. Annu Rev Cell Dev Biol

Parelho V, Hadjur S et al (2008) Cohesins functionally associate with CTCF on mammalian chromosome arms. Cell 132(3):422-433

Phair RD, Scaffidi P et al (2004) Global nature of dynamic protein-chromatin interactions in vivo: three-dimensional genome scanning, dynamic interaction networks of chromatin proteins. Mol Cell Biol 24(14):6393-6402

Rabut G, Ellenberg J (2005) Photobleaching techniques to study mobility, molecular dynamics of proteins in live cells: FRAP, iFRAP, FLIP. In: Goldman RD, Spector DL (eds) Live cell imaging: A laboratory manual. Cold Spring Harbor Laboratory Press, Cold Spring Harbor, NY, pp 101-126

Rollins RA, Morcillo P et al (1999) Nipped-B, a Drosophila homologue of chromosomal adherins, participates in activation by remote enhancers in the cut, Ultrabithorax genes. Genetics 152(2):577-593

Rollins RA, Korom M et al (2004) Drosophila nipped-B protein supports sister chromatid cohesion, opposes the stromalin/ Scc3 cohesion factor to facilitate long-range activation of the cut gene. Mol Cell Biol 24(8):3100-3111

Schmitz J, Watrin E et al (2007) Sororin is required for stable binding of cohesin to chromatin, for sister chromatid cohesion in interphase. Curr Biol 17(7):630-636

Sporbert A, Gahl A et al (2002) DNA polymerase clamp shows little turnover at established replication sites but sequential de novo assembly at adjacent origin clusters. Mol Cell 10 (6):1355-1365

Sprague BL, McNally JG (2005) FRAP analysis of binding: proper, fitting. Trends Cell Biol 15(2):84-91

Stedman W, Kang H et al (2008) Cohesins localize with CTCF at the KSHV latency control region, at cellular c-myc, H19/Igf2 insulators. EMBO J 27(4):654-666

Wallace JA, Felsenfeld G (2007) We gather together: insulators, genome organization. Curr Opin Genet Dev 17(5):400-407

Weber SA, Gerton JL et al (2004) The kinetochore is an enhancer of pericentric cohesin binding. PLoS Biol 2(9):E260

Wendt KS, Yoshida K et al (2008) Cohesin mediates transcriptional insulation by CCCTC-binding factor. Nature 451 (7180):796-801

Yeh E, Haase J et al (2008) Pericentric chromatin is organized into an intramolecular loop in mitosis. Curr Biol 18(2):81-90

Zhou Z, Elledge SJ (1993) DUN1 encodes a protein kinase that controls the DNA damage response in yeast. Cell 75 (6):1119-1127 